\title{
Impact of malaria interventions on child mortality in endemic African settings: comparison and alignment between LiST and Spectrum-Malaria model
}

Eline Korenromp ${ }^{1 *}$, Matthew Hamilton², Rachel Sanders², Guy Mahiané2, Olivier J. T. Briët ${ }^{3,4}$, Thomas Smith ${ }^{3,4}$, William Winfrey ${ }^{2}$, Neff Walker ${ }^{5}$ and John Stover ${ }^{2}$

\begin{abstract}
Background: In malaria-endemic countries, malaria prevention and treatment are critical for child health. In the context of intervention scale-up and rapid changes in endemicity, projections of intervention impact and optimized program scale-up strategies need to take into account the consequent dynamics of transmission and immunity.

Methods: The new Spectrum-Malaria program planning tool was used to project health impacts of Insecticide-Treated mosquito Nets (ITNs) and effective management of uncomplicated malaria cases (CMU), among other interventions, on malaria infection prevalence, case incidence and mortality in children 0-4 years, 5-14 years of age and adults. Spectrum-Malaria uses statistical models fitted to simulations of the dynamic effects of increasing intervention coverage on these burdens as a function of baseline malaria endemicity, seasonality in transmission and malaria intervention coverage levels (estimated for years 2000 to 2015 by the World Health Organization and Malaria Atlas Project). Spectrum-Malaria projections of proportional reductions in under-five malaria mortality were compared with those of the Lives Saved Tool (LiST) for the Democratic Republic of the Congo and Zambia, for given (standardized) scenarios of ITN and/or CMU scale-up over 2016-2030.

Results: Proportional mortality reductions over the first two years following scale-up of ITNs from near-zero baselines to moderately higher coverages align well between LiST and Spectrum-Malaria —as expected since both models were fitted to cluster-randomized ITN trials in moderate-to-high-endemic settings with 2-year durations. For further scale-up from moderately high ITN coverage to near-universal coverage (as currently relevant for strategic planning for many countries), Spectrum-Malaria predicts smaller additional ITN impacts than LiST, reflecting progressive saturation. For CMU, especially in the longer term (over 2022-2030) and for lower-endemic settings (like Zambia), Spectrum-Malaria projects larger proportional impacts, reflecting onward dynamic effects not fully captured by LiST.
\end{abstract}

Conclusions: Spectrum-Malaria complements LiST by extending the scope of malaria interventions, program packages and health outcomes that can be evaluated for policy making and strategic planning within and beyond the perspective of child survival.

Keywords: Malaria, Child health, Model, Prevention, Treatment, Health impact, Mortality, Africa

* Correspondence: ekorenromp@avenirhealth.org

${ }^{1}$ Avenir Health, PO box 2100, CH-1211 Geneva, Switzerland

Full list of author information is available at the end of the article 


\section{Background}

In malaria-endemic countries, malaria prevention and treatment are critical for child health. Between 2000 and 2015, malaria incidence rates fell $37 \%$ globally, and malaria mortality rates by $60 \%$, with even greater declines in Africa, the highest-burden region [1]. This was likely a combined result of improved malaria control and other factors independent of interventions [2]. Across all age groups in sub-Saharan Africa, malaria control interventions accounted for an estimated 70\% of the 943 million fewer malaria cases occurring between 2001 and 2015. For malaria deaths in children under-5, in 2012 the World Health Organization (WHO) had estimated that malaria prevention intervention scale-up over 20012010 had prevented 921,300 (uncertainty interval: $625,600-1,260,800$ ) child deaths (or $8.2 \%$ of the number in 2000) due to malaria across 36 malaria-endemic countries in Africa [3]. Impacts of improved case management, as well as mortality and morbidity impacts in older children and adults, are less certain.

As funding for malaria control has now plateaued, it is even more critical than before to prioritize interventions with the most impact, and determine and plan the optimal mix of interventions for each setting and time period - within the broader public health and health sector context. We developed a malaria strategic planning module in the Spectrum suite of policy models, to complement similar modules included for HIV/AIDS, tuberculosis and family planning, which are used by over 120 low and middle-income countries for estimation of burdens, trends, service needs and program impact [4-7]. The Spectrum-Malaria module extends the malaria impact modelling options available through the Lives Saved Tool (LiST) [3, 8, 9], by projecting impacts on not only mortality but also morbidity (malaria case incidence and prevalence of infection with Plasmodium falciparum (PfPR), for not only children 0-4 years but also children 5-14 years and adults. Besides Insecticide-Treated mosquito Nets (ITNs), Indoor Residual Spraying (IRS) and first-line treatment of malaria cases, also modelled in LiST, Spectrum-Malaria additionally projects the impact of severe case management of sever malaria cases, and seasonal malaria chemoprophylaxis for young children. Additional long-awaited refinements $[10,11]$ are the representation of dynamic effects of transmission and immunity over time and variation in impacts depending on baseline endemicity, intervention coverage levels - based on statistical summaries of simulations [12-14] performed in the dynamic model OpenMalaria $[15,16]$.

This article presents and compares projections of the impact on under-five mortality of ITNs and effective malaria case management, performed in SpectrumMalaria and in LiST, for a high-endemic and a lowendemic African setting. Differences and similarities are discussed with implications for interpreting modelling results for strategic planning. Options are discussed for updating and aligning both models and their input data and assumptions, and for operating both models in combination to cover the fullest set of interventions relevant for comprehensive program planning.

\section{Methods}

Projections were made for the Democratic Republic of the Congo (DRC), as an example of a setting with stable high endemicity, and Zambia as an example of a setting with lower endemicity, over a 15-year horizon, and under-5 mortality impacts were compared between LiST and Spectrum-Malaria.

\section{Spectrum-Malaria projections}

The Spectrum-Malaria model of 15th June 2016 (version 5.45 Beta 4) was used, detailed elsewhere [12, 14]. This includes statistical regression models that predict the proportional reduction in PfPR, case incidence and malaria mortality, for a given change in intervention coverage, fitted to simulations of such intervention scenarios in the dynamic transmission model OpenMalaria [13]. Spectrum thus provides a simple, quick calculation tool for program planners that captures most of the dynamics of the full OpenMalaria research model. Interventions included were: ITNs, IRS, effective management of uncomplicated malaria cases (CMU) and of severe cases, and seasonal malaria chemoprophylaxis for children 359 months.

For simulations underlying Spectrum's statistical estimates of ITN impacts, the effectiveness of ITNs (and IRS) in the OpenMalaria model had been calibrated [13] to fit the observed 2-year impacts on under-five mortality, case incidence and parasite infection prevalence from three ITN trials in Kenya and Ghana [17], that were also the basis for mortality effectiveness assumptions in LiST [9]. Effectiveness of management of uncomplicated malaria cases was modeled assuming a $100 \%$ cure rate for malaria patients complying with an effective antimalarial treatment, e.g. with an artemisinin-based combination therapy (ACT) [18].

Malaria mortality rates at 2014 and 2015 (and preceding years) were taken from the WHO's official national estimates of November 2015 [1] for children under-5 versus older age groups. Baseline (2000-2015) data for the impact modifiers, seasonality in malaria transmission, and PfPR in children 2-9 years, were taken from estimates by the Malaria Atlas Project $[2,19]$-aggregated from $5 \times 5$ $\mathrm{km}^{2}$ pixel level estimates into first administrative level (Admin1) units (i.e. states or provinces). National-level impacts are computed by Spectrum-Malaria by aggregating burden and impact projections for each province in a country, thus taking account of (part of the) sub- 
national variation in impact determinants. The WHO's national-level mortality estimates were distributed over Admin 1 units by assuming a similar proportional distribution as estimated by the Malaria Atlas Project for malaria case incidence $[2,19]$.

For projections over 2016-2030, Spectrum applied proportional impacts predicted by regression models fitted to the average outcomes over years 1-3 after intervention scale-up from OpenMalaria simulations [13] over 2016-2021; for 2022-2030 Spectrum applied the proportional impacts from regression models fitted to average OpenMalaria simulation outcomes over years 810 after scale-up [13]. Spectrum applies these impact functions with a one-year time lag from intervention scale-up to start of impact, thus the projected burdens in 2016 reflected the effect of intervention coverage changes from 2014 to 2015 , and onwards (Table 1).

\section{LiST projections}

LiST was used in the version 'Spectrum 5.43 beta 1' of May 2016. Outputs analyzed were the cause-specific deaths in children under five years (i.e. 0-59 months) of age. Allcause neonatal and post-neonatal mortality rates at 2014 and 2015 (and preceding years) were taken from estimates by the United Nations Inter-Agency Group for Child Mortality Estimation as of 2015 [20] (Table 2). The corresponding malaria mortality rates were derived by applying the proportion of post-neonatal under- 5 deaths due to malaria (among eight other causes) from the WHO in the version as of October 2015 (http://www.who.int/healthinfo/ global_burden_disease/estimates/en/index3.html) and [1].

Effectiveness of ITNs and/or IRS (with the coverage definitions detailed in Table 3), was estimated and implemented as children 1-59 months living in households protected by ITNs and/or IRS having a 55\% lower risk of malaria-attributable death [9]. Treatment of Plasmodium falciparum malaria cases (without distinction between uncomplicated and severe cases) with artemisinin combination therapy (ACT) was assumed to reduce malaria mortality in children 1-23 months by $99 \%$ (range: 94-100\%), and in children $24-59$ months by $97 \%$ (range: 86-99\%) [21].

\section{Standardized intervention scale-up and scale-down scenarios}

For the current model comparisons, baseline coverages and intervention scale-up targets for ITNs and CMU were standardized between LiST and Spectrum-Malaria (Table 3, row labeled 'Coverage-standardized, DRC \& Zambia'), and between the two countries, so as have a similar extent of scale-up from baseline to target level in both countries. Coverage values were set in the range of default coverage values assumed by the two models for the two countries, as explained in the following two paragraphs and with precise annual values shown in Additional file 1.

For vector control, LiST uses a combined coverage metric combining protection by ITNs and/or IRS, defined as the proportion of children under- 5 who live in a household owning one or more ITNs, and/or in a house that has been sprayed with IRS within the past 12 months. Spectrum-Malaria uses as coverage metrics: for ITNs, the proportion of the population of all ages who slept under an ITN the last night; and for IRS, the proportion of the population of all ages who live in a house sprayed with IRS within the past 12 months. The standardized coverage assumptions we used for projections and comparisons were: for LiST, 70\% ITN/IRS protection at 2014 and 2015 (close to the LiST default values of $70 \%$ for DRC and $74.7 \%$ for Zambia), increasing to $98 \%$ as the maximum target level (at 2016, or at 2020 with linear increase over 2016-2020); for Spectrum-Malaria, the standardized coverage assumption (judged most similar to standardized coverage in LiST) was 51\% ITN usage at 2014 and 2015 (slightly below the Spectrum-Malaria default values of $55 \%$ for DRC and $68.8 \%$ for Zambia), increasing to $70 \%$ as maximum target level. We considered $51 \%$ and $70 \%$ usage to be similar to $77 \%$ and $98 \%$ of households owning one or more ITNs, based on ratios between these metrics in DRC, Zambia and other stable malaria-endemic African countries observed in national household surveys ([2224] and Additional file 2), and therefore we consider these standardized coverage values optimal for the purpose of comparing impact projections despite the different coverage metrics in the two models. In Spectrum-Malaria, IRS coverage was kept constant throughout 2014-2030, so that IRS coverage did not have any projected impact.

For malaria case management, Spectrum-Malaria distinguishes between uncomplicated cases and severe cases, but LiST does not. We compared management of uncomplicated cases from Spectrum-Malaria with overall case management from LIST. For these respective interventions, both models use as input the coverage of child fevers treated with an ACT, as observed in national household surveys: most often, Demographic and Health Surveys (DHS) [25], Multiple Indicator Cluster Surveys (MICS) [26] and Malaria Indicator Surveys (MIS) [27]. LiST inputs new survey data points individually and continually as these survey data are released, while Spectrum-Malaria pre-loaded a multi-country dataset of survey-based coverage estimates for 2005, 2010 and 2015 , in which survey data were adjusted and extrapolated in a standardized way to years and countries without surveys [28]. In Spectrum-Malaria for most African countries including DRC and Zambia, less than $8 \%$ of cases are severe $[12,14]$, so the difference in denominators (uncomplicated cases versus all malaria cases) should not 
Table 1 Key structural and methodological differences between LiST and Spectrum-Malaria

\begin{tabular}{ll}
\hline Aspect & LiST \\
\hline Health outcomes considered, that are & - Malaria-attributable, other-cause and \\
impacted by malaria interventions & all-cause mortality in children 0-4 years \\
& (separately for neonatal, 1-12 months and \\
& $13-59$ months sub-groups) \\
& - Maternal deaths \\
& - Stillbirths \\
& - Vector control (IRS and/or ITNs) \\
Interventions modelled & Case management, uncomplicated malaria \\
& cases \\
& - Intermittent Preventive Therapy for \\
& pregnant women
\end{tabular}

Determinants of impact of malaria intervention scale-up

Determinants of impact, for a given coverage increase

Synergy or saturation across interventions?

Saturation of incremental impacts at higher coverages?

Time path of impact

Basis and source of coverage-impact relationship: ITNs

Basis and source of coverage-impact relationship: case management
Proportional reduction from baseline burden level, for a given proportional increase in intervention coverage - same within and across all countries

Fixed effectiveness value (for ITNs and CMU: from sources below), for all years and all countries [48]
Spectrum-Malaria

- Malaria-attributable mortality and case

incidence, in 0-4 years, 5-14 years and

$15+$ years;

- Plasmodium falciparum infection

prevalence (PfPR), in children 2-9 years

- ITNS

- IRS

- Case management, uncomplicated malaria cases (CMU)

- Case management, severe malaria cases

- Seasonal Malaria Chemoprophylaxis (SMC)

in children 3-59 months

Proportional reduction from baseline burden level, varying with baseline endemicity (PfPR), seasonality in malaria transmission, and baseline and scale-up coverage levels of ITNs, IRS, CMU and SMC, which act in interaction), calculated at province-level and then aggregated to national level [13]

- Baseline malaria endemicity (PfPR in 2-9 years),

- Seasonality in malaria transmission,

- Baseline and scale-up coverage levels of other malaria interventions that have time-dynamic impacts (ITNs, IRS, SMC, and management of uncomplicated cases), and their variations between provinces, within and across countries [13].

Yes [13].

Yes [13].

Nor Yes [13].

No variation over time: impact is immediate from the year of scale-up; the post-intervention mortality level stays constant thereafter until coverage changes again

- Impact modelled with a 1-year lag after intervention scale-up;

- Separate impact functions for short term (years 2-6 after scale-up) and long term (years 7-15 after scale-up) [12, 14].

United Nationals Child Health Epidemiology Reference Group (CHERG), meta-analysis of randomized ITN trials [9]

Meta-analyses of published observational studies and a previous Delphi estimate [21]
Dynamic transmission model simulations for a wide range of sub-Sahara-Africa like scenarios, varying in endemicity, seasonality and baseline intervention coverages, performed in the OpenMalaria model summarized in multi-variate statistical models [13]. OpenMalaria intervention effectiveness assumptions were in turn based on meta-analysis of randomized ITN trials [9], and review of observational treatment impact studies [18, 49]

Differences in data sources, intervention coverage definitions and country baseline parameter values are described in Tables 1 and 2

invalidate the comparison of their impacts. For the current projections, coverage in both DRC and Zambia was set at $10 \%$ for 2014 and 2015 , increasing to $40 \%$ as maximum target level (again reached either in 2016, or in alternative scenarios by 2020 with linear scale-up over 2016-2020).

Using these target coverage levels for ITNs and CMU, we modelled and compared the following 6 scale-up scenarios:

- Scaling-up ITN to the stated target coverage;

- Scaling-up CMU to the stated target coverage;
- Scaling-up ITNs and CMU to the stated respective target coverages, in parallel; with for each of these 3 intervention targets: either scale-up at once realized in 2016 and maintained throughout 2030, or a linear scale-up starting in 2016 with the target coverage level reached by 2020 and then maintained until 2030.

In addition, to show the coverage-to-impact relationship over a wide coverage range, including the effect of scaling-down coverage to below the 2014-2015 baseline 
Table 2 Parameters and input data used by LiST and Spectrum-Malaria in projections for DRC and Zambia

\begin{tabular}{|c|c|c|c|c|c|c|}
\hline \multirow{2}{*}{$\begin{array}{l}\text { Parameter (2015, unless } \\
\text { otherwise indicated) }\end{array}$} & \multicolumn{2}{|l|}{ DRC } & \multicolumn{2}{|l|}{ Zambia } & \multicolumn{2}{|l|}{ Data sources \& definitions } \\
\hline & Spectrum-Malaria & LiST & Spectrum-Malaria & LiST & Spectrum-Malaria & LiST \\
\hline Population at malaria risk ${ }^{\mathrm{a}}$ & $97.1 \%$ & $91 \%$ & $100 \%$ & $98 \%$ & $\begin{array}{l}\text { All-age population living where PfPR among } \\
2-9 \text { years }>0[2,19]\end{array}$ & $\begin{array}{l}\text { Women exposed } \\
\text { to } P \text {. falciparum } \\
\text { malaria [50] }\end{array}$ \\
\hline $\begin{array}{l}\text { Population } 0-4 \text { years (including } \\
\text { children living not at } \\
\text { malaria risk) }\end{array}$ & $12,373,927$ & $13,682,392$ & $2,848,069$ & $2,888,817$ & \multicolumn{2}{|l|}{ United Nations Population Division [51] } \\
\hline $\begin{array}{l}\text { Index for seasonality in malaria } \\
\text { transmission }\end{array}$ & 0.36 & NA & 1.64 & NA & \multicolumn{2}{|l|}{$\begin{array}{l}\text { Coefficient of variation in EIR over a year, } \\
\text { defined as the standard deviation divided } \\
\text { by the year-average of monthly EIR [34]; } \\
\text { country estimates from Malaria Atlas } \\
\text { Project }[2,19]\end{array}$} \\
\hline $\begin{array}{l}\text { Prevalence of } P \text {. falciparum } \\
\text { infection in children 2-9 years, } \\
\text { average over 2000-2002 }\end{array}$ & $64 \%$ & NA & $35 \%$ & NA & \multicolumn{2}{|l|}{ Malaria Atlas Project [19] } \\
\hline $\begin{array}{l}\text { Malaria deaths in children } \\
0-59 \text { months (\% of all-cause } \\
\text { deaths) }\end{array}$ & 33,038 & $47,473(16 \%)$ & 2734 & $2723(5.9 \%)$ & $\begin{array}{l}\text { WHO (http://www.who.int/healthinfo/ } \\
\text { global_burden_disease/estimates/en/ } \\
\text { index3.html and [1]) }\end{array}$ & WHO \\
\hline All-cause under- 5 deaths & NA & 298,200 & NA & 45,916 & NA & $\begin{array}{l}\text { UN Inter-Agency } \\
\text { Group for Child } \\
\text { Mortality Estimation [20] }\end{array}$ \\
\hline Malaria deaths in 5-14 years & 3258 & NA & 2226 & NA & \multirow{2}{*}{$\begin{array}{l}\text { WHO [1]; share of 5-14 versus } 15+\text { years as } \\
\text { for malaria cases }[2,19]\end{array}$} & \multirow[t]{2}{*}{ NA } \\
\hline Malaria deaths in $15+$ years & 2936 & NA & 2074 & NA & & \\
\hline $\begin{array}{l}\text { Malaria cases i.e. disease episodes } \\
\text { in } 0-4 \text { years }\end{array}$ & $8,231,156$ & NA & $1,188,935$ & NA & $\begin{array}{l}\text { WHO [1]; the share of } 0-4 \text { years in WHO's } \\
\text { all age estimate taken from Malaria Atlas } \\
\text { Project }[2,19]\end{array}$ & NA \\
\hline
\end{tabular}

Abbreviations: EIR Entomological Inoculation Rate, LiST Lives Saved Tool, DRC Democratic Republic of the Congo, MAP Malaria Atlas Project, P. falciparum Plasmodium falciparum, NA not available

aThe population at risk of malaria does not influence impact calculations, but it is used in the OneHealth Tool costing as the 'Population in Need' (PIN) that would need to get various services like ITNs, IRS spraying, etc. (Equation for number of services: Target Population * PIN * Coverage)

level, several scale-down scenarios were modelled (details in Additional file 1), with each coverage change implemented at once in 2016 and sustained until 2030.

\section{Health outcomes and time horizons evaluated}

Impacts were compared as proportional reductions in under-5 mortality numbers, so that some small differences in baseline mortality rates between the two models (Table 2) did not affect the comparison.

We evaluated mortality impacts over 2016-2030, the horizon considered in Spectrum-Malaria (while LiST does not specify a specific horizon of validity). Some comparisons focused on either 2020 or 2025, as indicative of shorter-term impacts versus longer-term impacts, respectively, being modelled through distinct statistical impact functions in Spectrum-Malaria [13].

\section{Results}

\section{Scaling-up ITN and CMU from 2015 coverage levels}

In both LiST and Spectrum-Malaria, increasing CMU coverage from $10 \%$ to $40 \%$ has larger impact than increasing ITN coverage from $77 \%$ ownership or $51 \%$ usage to $98 \%$ ownership or $70 \%$ usage (Figure 1).

In LiST, intervention scale-up reduces mortality to a new post-intervention level within the same year as the increased intervention coverage; this new mortality rate is maintained over next years and annual numbers of deaths reflect this rate multiplied by the (growing) population size. In Spectrum-Malaria, intervention scale-up starting in 2016 results in a reduced mortality rate over 2017-2021, with a further reduced mortality rate over 2022-2030 reflecting the long-term transmission dynamic effect. For ITNs, proportional under- 5 mortality reductions following scale-up are somewhat larger in LiST than in Spectrum-Malaria, for both DRC and Zambia in the short-term over 2016-2021 (Fig. 2, left). Over 2022-2030, proportional ITN impacts are similar between both models for Zambia, but still larger in LiST for DRC (Fig. 2, right).

For CMU, in contrast, Spectrum-Malaria predicts larger proportional mortality reductions than LiST, for both countries, across 2016-2030 and throughout the coverage range of $0-100 \%$, but especially so for lower-endemic Zambia and for the longer (2022-2030) time horizon.

For both interventions, LiST projects identical proportional impacts for a given coverage increase in DRC and Zambia (Fig. 2 \& Table 4), and across all other countries (not shown). Spectrum-Malaria, in contrast, projects larger proportional burden reductions for the lowerendemic Zambia, compared to the higher-endemic DRC.

Scaling-down ITN and CMU from 2015 coverage levels For scenarios decreasing ITN coverage to below the 2015 actual coverages (which were already high in both 
Table 3 Intervention coverage at 2015 in DRC and Zambia, and two coverage-standardized country variants modelled

\begin{tabular}{|c|c|c|c|c|}
\hline & Module & ITN coverage & IRS coverage & Malaria case management \\
\hline \multirow[t]{2}{*}{$\overline{D R C}$} & $\begin{array}{l}\text { Spectrum- } \\
\text { Malaria }\end{array}$ & Usage: 55\% & $0.27 \%$ & $6.6 \%$ \\
\hline & LiST & ITN owning and/or IRS-sprayed: 70\% & & $1.7 \%$ \\
\hline \multirow[t]{2}{*}{ Zambia } & $\begin{array}{l}\text { Spectrum- } \\
\text { Malaria }\end{array}$ & Usage: $68.8 \%$ & $35.6 \%$ & $44.1 \%$ \\
\hline & LiST & ITN owning and/or IRS-sprayed: $74.7 \%$ & & $18.4 \%$ \\
\hline \multirow[t]{2}{*}{$\begin{array}{l}\text { Coverage- } \\
\text { standardized, } \\
\text { DRC \& Zambiab }\end{array}$} & $\begin{array}{l}\text { Spectrum- } \\
\text { Malaria }\end{array}$ & $\begin{array}{l}\text { - 51\% ITN usage at } 2014 \text { \& 2015; } \\
\text { - } 70 \% \text { ITN usage as target for 2016-2020; } \\
\text { - IRS: } 0.28 \% \text { throughout } 2014-2030 .\end{array}$ & & $\begin{array}{l}\cdot 10 \% \text { at } 2014 \& 2015 \\
\cdot 40 \% \text { as target for } 2016-2020\end{array}$ \\
\hline & LiST & $\begin{array}{l}\text { - } 77 \% \text { ITN owning and/or IRS sprayed } \\
\text { at } 2014 \text { \& 2015; } \\
\text {. } 98 \% \text { as target for 2016-2020 }\end{array}$ & & $\begin{array}{l}\text { - } 10 \% \text { at } 2014 \text { \& } 2015 \\
\text { - } 40 \% \text { as target for } 2016-2020 \text {. }\end{array}$ \\
\hline \multirow[t]{2}{*}{$\begin{array}{l}\text { Data source \& } \\
\text { definition }\end{array}$} & $\begin{array}{l}\text { Spectrum- } \\
\text { Malaria }\end{array}$ & $\begin{array}{l}\text { WHO/MAP } 2015 \text { [24], \% of national } \\
\text { population sleeping under an ITN } \\
\text { (including areas with PfPR }=0 \text { ). }\end{array}$ & $\begin{array}{l}\text { WHO } 2015[1], \% \text { of national } \\
\text { population protected (all ages, } \\
\text { including areas with PfPR }=0 \text { ) }\end{array}$ & $\begin{array}{l}\text { ACT treatment of RDT-positive } \\
\text { (and RDT-negative) fevers in } \\
\text { children 0-4 years [28], applied to } \\
\text { uncomplicated malaria cases in all } \\
\text { age groups }\end{array}$ \\
\hline & LiST & \multicolumn{2}{|c|}{$\begin{array}{l}\text { Households owning } \geq 1 \text { ITNs or protected by IRS as measured } \\
\text { in the most recent national-representative household survey } \\
\text { (usually MIS, MICS or DHS) }^{\mathrm{a}}\end{array}$} & $\begin{array}{l}\text { Children } 0-4 \text { years with a fever } \\
\text { treated within } 48 \mathrm{~h} \text { of fever onset } \\
\text { with an artemisinin-containing } \\
\text { compound i.e. ACT as measured } \\
\text { in the most recent national- } \\
\text { representative household survey } \\
\text { (usually MIS, MICS or DHS) }^{\text {a }}\end{array}$ \\
\hline
\end{tabular}

Abbreviations: ACT Artemisinin-based Combination Therapy, DHS Demographic and Health Survey, DRC Democratic Republic of the Congo, ITN Insecticide-treated mosquito net, IRS Indoor Residual Spraying, LiST Lives Saved Tool, MAP Malaria Atlas Project, MICS Multiple Indicator Cluster Survey, MIS Malaria Indicator Survey, SSA sub-Saharan Africa, Swiss TPH Swiss Institute of Tropical and Public Health, PfPR Plasmodium falciparum parasite prevalence rate

${ }^{a}$ LiST uses the measured values for years in which surveys occurred, and applies linear interpolation between measured points. For years after the last survey in a country, LiST assumes that coverage is constant

${ }^{\mathrm{b}}$ Precise annual coverages for each intervention, scenario, and model are shown in Additional file 1

DRC and Zambia, and in our coverage-standardized variants of these countries), Spectrum-Malaria predicts a similar mortality increase as LiST for DRC over the first five years after ITN scale-down (represented as a drop from $51 \%$ to $12.5 \%$ utilization, or from $77 \%$ to $18 \%$ ownership), followed by a further mortality rise compared to LiST from the 7th year following scale-down (Fig. 3). For Zambia, in the short-term (over 2016-2021) and especially the longer-term (over 2022-2030), the projected mortality rise due to ITN coverage decrease is larger in Spectrum-Malaria than in LiST.

\section{Non-linearity in the coverage-impact relationship}

We compared mortality impacts for a given coverage increase or decrease, over the full $0-100 \%$ range of possible coverages and between the countries, as comparative proportional mortality reductions relative to the mortality level under a constant-coverage scenario, at the year 2020. In LiST, the effect of a given coverage increase is calculated as a simple multiplication of (reduced) relative risks that are linear with the coverage increase, identically for all countries, as shown in Fig. 4, with identical lines for DRC and Zambia. Spectrum-Malaria, in contrast, shows larger proportional impacts of both ITN and CMU for the lower-endemic Zambia (steeper curves in Figs. 4b and d) than for higher-endemic DRC. Spectrum-Malaria furthermore shows an important saturation effect apparent at coverage levels of around $50 \%$ or higher, for both interventions: adding 20 percentage points ITN or CMU coverage from a moderately high baseline level of e.g. $50 \%$ coverage does not produce as large a mortality reduction as adding 20 percentage point coverage from a low baseline level of e.g. $10 \%$.

\section{Interaction between ITNs and CMU}

A non-linearity that is captured in Spectrum-Malaria but not in LiST is interaction between interventions, for example between ITNs and CMU. In LiST, the proportional impacts of concurrent ITN/IRS and CMU scaleup equate the product of the proportional impacts of the two interventions when implemented each in isolation (Table 4). In Spectrum-Malaria, in contrast, for both DRC and Zambia, concurrent scale-up to high coverage levels for ITNs and CMU simultaneously entails a slight saturation in the overall impact, with the overall proportional reduction slightly less than the product of the corresponding relative reductions achieved by the two interventions when each applied alone. 


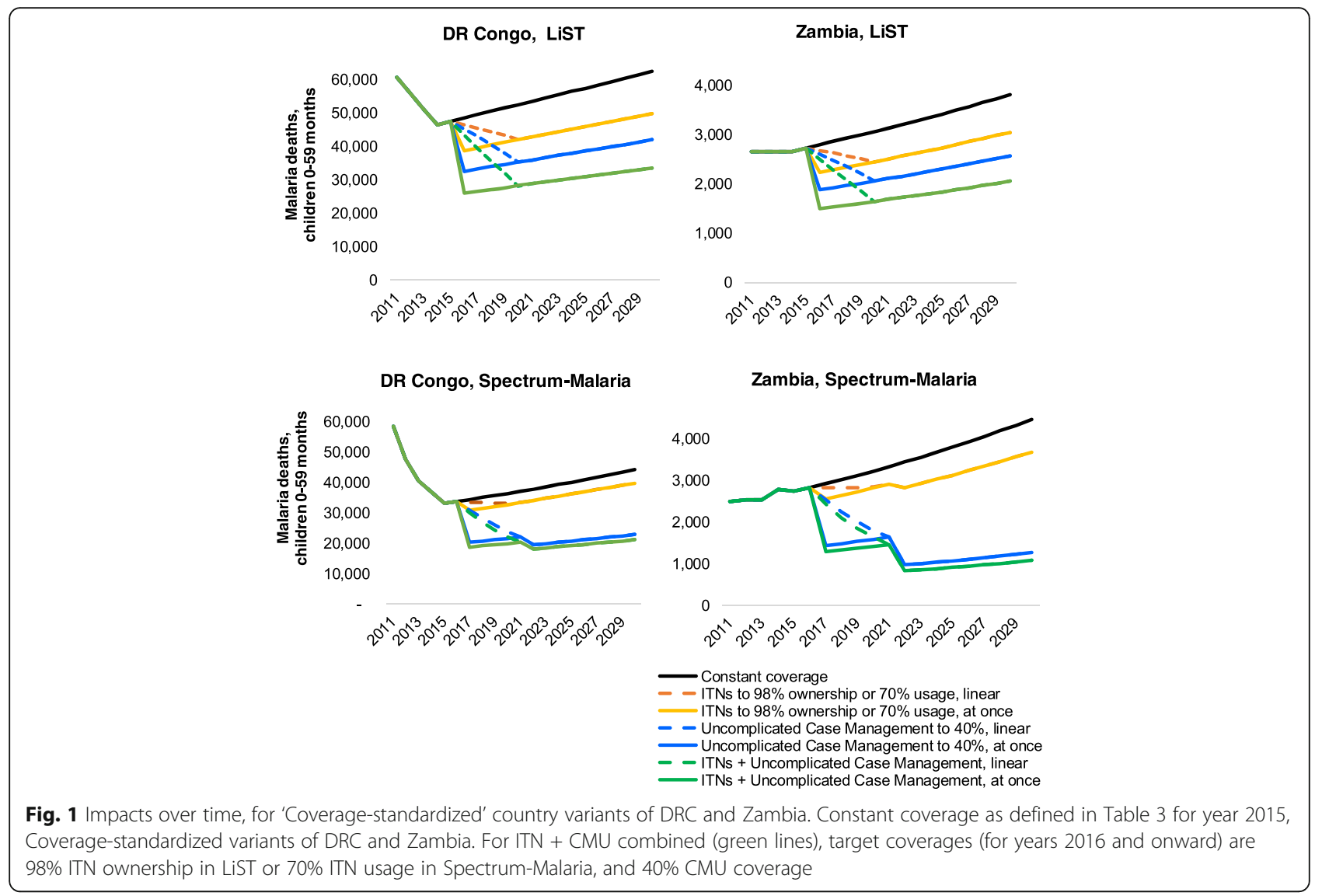

\section{Discussion}

The presented projections of impacts of ITNs and CMU on malaria mortality from two models show how Spectrum-Malaria usefully corroborates and complements LiST, in projecting impacts of key malaria interventions including their variation over time with some dynamic transmission effects materializing from six years after scale-up, variation by baseline endemicity and as a function of baseline coverage levels, and dynamic interactions between interventions. Overall, Spectrum-Malaria broadly supports the magnitude of mortality impacts of ITNs and CMU projected by LiST [3] and several retrospective impact evaluations $[2,29,30]$ for falciparum-endemic African settings. The similar proportional mortality reductions over the first two years following scale-up of ITNs from near-zero baselines to moderately higher coverages are as expected, since both models were validated on cluster-randomized ITN trials in moderate-to-high-endemic settings with a 2-year duration. The ITN-related mortality reductions projected are also in line with

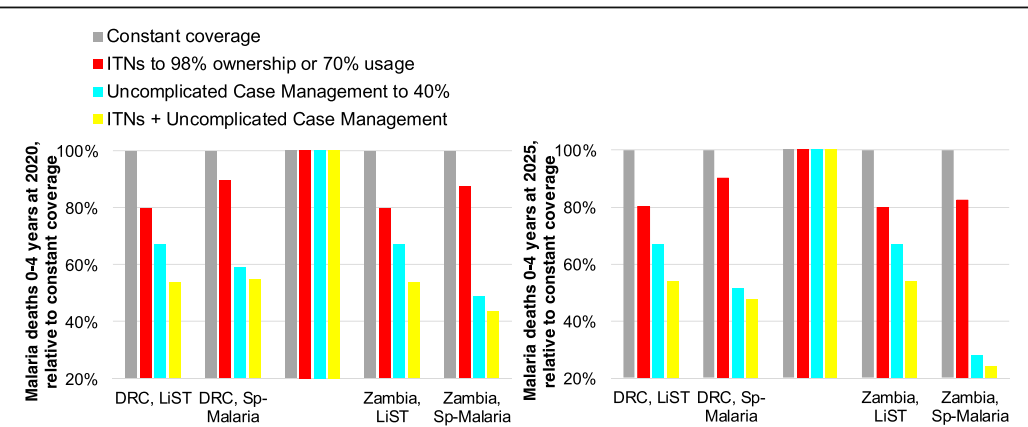

Fig. 2 Deaths in 0-4 year-olds, relative to constant-coverage scenario, (left) 2020 and (right) 2025. Constant coverage as defined in Table 3 for year 2015, Coverage-standardized variants of DRC and Zambia. For ITN + CMU combined (yellow bars), target coverages (for years 2016 and onward) are 98\% ITN ownership in LiST or 70\% ITN usage in Spectrum-Malaria, and 40\% CMU coverage 
Table 4 Relative under-5 malaria mortality levels after simultaneous scale-up of CMU and ITNs

\begin{tabular}{|c|c|c|c|c|}
\hline \multirow[t]{2}{*}{ Scale-up scenario } & \multicolumn{2}{|l|}{ LiST } & \multicolumn{2}{|c|}{ Spectrum-Malaria } \\
\hline & $\mathrm{DRC}$ & Zambia & $\mathrm{DRC}$ & Zambia \\
\hline $\begin{array}{l}\text { ITN 77\% ownership or 51\% usage; Uncomplicated Case Management 10\% } \\
\text { (i.e. constant coverage scenario of Figs. 1, } 2 \text { and 3) }\end{array}$ & $100 \%$ & $100 \%$ & $100 \%$ & $100 \%$ \\
\hline ITN $98 \%$ ownership or $70 \%$ usage (single intervention) & $80 \%$ & $80 \%$ & $90 \%$ & $88 \%$ \\
\hline Uncomplicated Case Management 40\% (single intervention) & $67 \%$ & $67 \%$ & $59 \%$ & $49 \%$ \\
\hline $\begin{array}{l}\text { ITN } 98 \% \text { ownership or } 70 \% \text { usage \& Uncomplicated Case Management 40\% } \\
\text { (two interventions concurrently) }\end{array}$ & $53.8 \%$ & $53.8 \%$ & $54.7 \%$ & $43.8 \%$ \\
\hline $\begin{array}{l}\text { Product of Relative mortality levels after ITN scale-up (to } 98 \% \text { ownership or } \\
70 \% \text { usage) alone, and after scale-up of Uncomplicated Case Management } \\
\text { (to } 40 \% \text { ) alone }\end{array}$ & $53.9 \%$ & $53.8 \%$ & $53.2 \%$ & $42.8 \%$ \\
\hline
\end{tabular}

The results percentages reflect the mortality level at 2020 in the scale-up scenario, relative to the mortality level at 2020 in the scenario with coverages held constant at 2015 levels. Each coverage scale-up was implemented as an immediate coverage increased from 2015 to 2016 , and maintained over 2016-2030. ITN ownership indicates coverage level projected in LiST; ITN usage indicates coverage level projected in Spectrum-Malaria

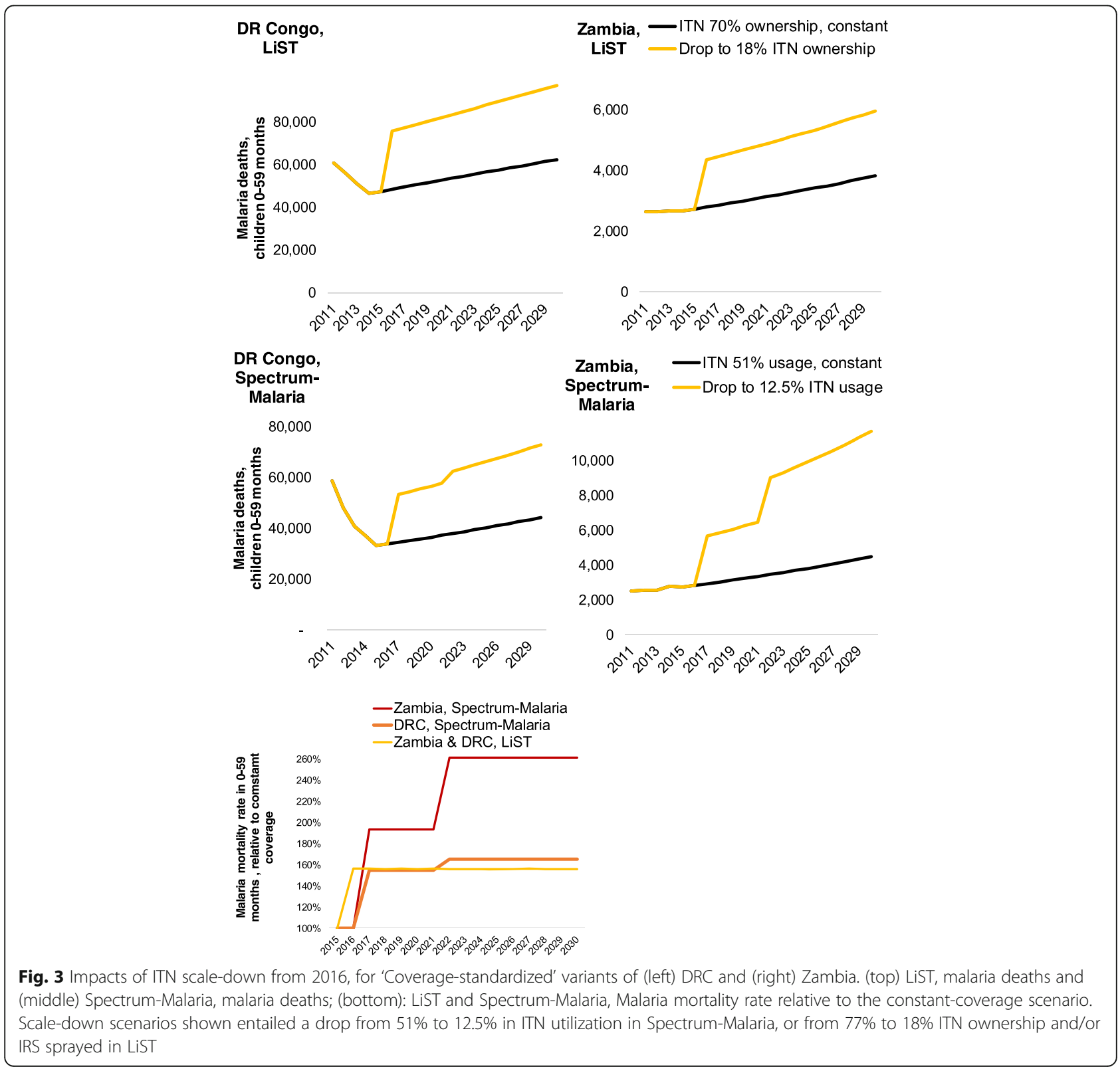



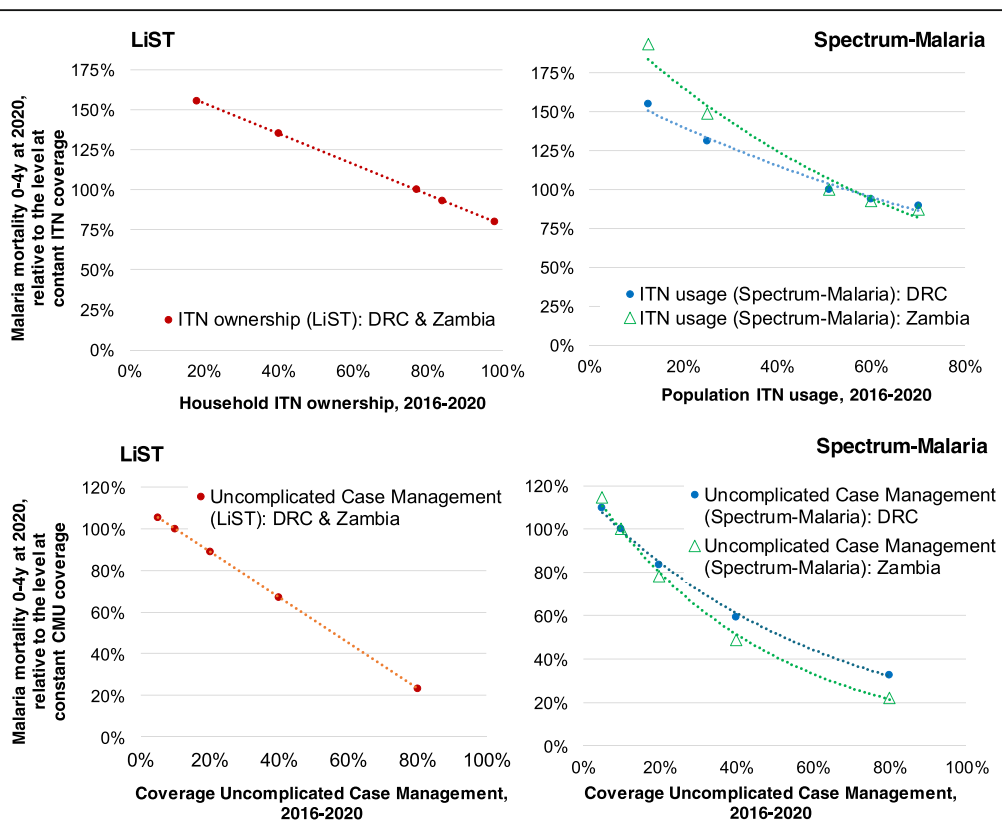

Fig. 4 Non-linearities in mortality impacts of ITNs and CMU, following scale-up or scale-down at 2016. The y-axes express the mortality level at 2020, in the scale-up or scale-down scenario, relative to the mortality level projected at 2020 under 'constant' coverages as shown in Figs. 1, 2 and 3: CMU coverage is kept constant at 10\%, ITN household ownership at 77\% (in LIST) and ITN usage at 51\% (in Spectrum-Malaria); the mortality level at these coverage levels are each displayed as 100\%, so as to allow displaying both countries within the same chart despite their differing malaria mortality levels

magnitudes of mortality decline associated with the increased malaria donor funding over the 2000s, which had been allocated primarily to ITN distribution programs and improved case management $[31,32]$.

In comparison, a recent assessment of impacts expected over 2015-2030 from scaling-up malaria control according to the WHO's Global Technical Strategy, using the Imperial College London malaria transmission model, projected a possible reduction in malaria mortality rates of $40 \%$ (across all ages) from 2010 to 2030 across 80 countries with sustained stable malaria transmission in 2010 [33]. This would seem to be a smaller impact than projected by LiST and especially Spectrum-Malaria for the combined scale-up of ITN and CMU in DRC and Zambia (Fig. 2). Strict quantitative comparison should rather be pursued country by country and age group by age group, for standardized intervention packages and coverage increments, as recently done with OpenMalaria, the Imperial College London model and two other dynamic models for the child health impacts of RTS,S vaccination [34]. That comparison illustrated that uncertainties about malaria pathogenesis as a function of acquired immunity, and the resulting age distribution in malaria burdens, especially in low-transmission settings, leave a considerable margin of uncertainty about the magnitude of (proportional and absolute) health impacts of malaria control, and about effectiveness rankings among interventions. In this context, the projection results that we presented for DRC and Zambia in fact seem quite consistent between LiST and Spectrum-Malaria.

Importantly, the Spectrum-Malaria projections suggest that the gains expected from ongoing ITN scale-up toward universal coverage - as targeted now by the WHO's Global Technical Strategy for Malaria 2016-2030 - may be somewhat smaller than predicted by LiST, while the potential impact of CMU may be larger. In drawing national strategic plans for malaria control, program planners should, however, consider the modelled ranking of impacts alongside mapping of the cascades of services, activities and inputs needed to achieve a given coverage level and increase - which for ITNs (through vertical distribution campaigns) may be easier than for CMU (which typically requires a broader health systems strengthening). Tellingly, of the 663 million malaria cases averted in subSaharan Africa due to malaria control interventions over 2000-2015, it was estimated that $69 \%$ were averted due to use of ITNs, and only $21 \%$ due to ACT-based treatment, and $10 \%$ due to IRS [2]. Spectrum-Malaria can support comprehensive impact and cost-effectiveness evaluation through its One Health Tool module, which links with impacts projected through LiST and the Spectrum-Malaria module.

The structural differences between Spectrum-Malaria and LiST (Table 1) underlie several important differences in impact results, besides different (generally larger) longterm impacts compared to short-term population level 
impacts. First, Spectrum-Malaria projects proportionally larger burden reductions for settings with lower baseline burdens. This was evident in the current projections as larger proportional mortality reductions for Zambia compared to DRC, and it applies to other settings and other malaria-related health outcomes as well, being a function of statistical relationships driving impact projection in Spectrum-Malaria, which include a negative coefficient for PfPR, so that settings with higher PfPR will systematically have lesser proportional burden reductions for any given coverage increase [13]. Of note, while this pattern was illustrated here as a difference between Zambia and $\mathrm{DRC}$, the endemicity effect is modelled in SpectrumMalaria at the sub-national level (of Admin1 units, i.e. first sub-national level), so that within Zambia, within DRC or within any other country, the proportional health impacts are larger in lower-endemic provinces or states than in those with higher endemicity. This modelled pattern is consistent with observations from ITN trials [17] and with models of the dynamics induced by various malaria interventions [34-37]. In contrast, absolute health gains -in terms of cases and deaths averted for a given coverage increase - are generally larger for higher-endemic settings (e.g. DRC more than Zambia), due to their larger baseline burden compared to lower-endemic settings.

Second, more influentially, in Spectrum-Malaria the incremental impacts for a given coverage percentage increase saturate at higher coverages. LiST, in contrast, by default calculates impacts fully linearly with coverage increases throughout the $0-100 \%$ coverage range. And a special option in LiST called 'transmission herd effect' allows users to even amplify impacts of ITN and/or IRS protection by specifying proportions of children who are themselves uncovered by ITN and/or IRS to still be protected from malaria - but there are no default assumptions for this effect, and the herd effect option cannot model negative (saturating) effects of saturating ITN or IRS coverage.

Third, in Spectrum-Malaria, the combined impact of simultaneous scale-up of multiple interventions does not necessarily equate the product of the individual interventions' proportional impacts (as in LiST), but this can be less due to saturation effects, as shown for concurrent ITN and CMU scale-up in DRC and Zambia (Figure 3). In other settings, notably higher-endemic settings with lower baseline and target coverages (not shown), SpectrumMalaria projects synergy between interventions, whereby combinations of interventions reduce transmission and endemicity more strongly, and the incremental effect of additional interventions then amplifies the overall effect beyond the contributions of single interventions [13]. These saturation and synergic effects reflect the dynamics of malaria control, as simulated in OpenMalaria [38] and emulated through Spectrum's statistical impact functions [13].
Fourth (less relevant to the child health perspective and comparison with LiST, but important for overall infectious disease and health sector planning) SpectrumMalaria projects important impacts of malaria control on morbidity and mortality in children above five years and adults. These older age groups have much lower shares in malaria morbidity and mortality burdens, which malaria intervention coverage scale-up would reduce by near-similar proportions as shown here for children under-5 - but with some rebounds in longer-term reflecting declining acquired immunity in cohorts of people benefiting from enhanced malaria protection [13]. As a result, Spectrum-Malaria, like the dynamic transmission models that informed it, predicts that enhanced malaria control will progressively shift malaria burden to older ages and result in a more homogeneous distribution of malaria over age [39] (as already seen in lower-endemic settings outside of Africa), heightening the importance of including over-fives in impact evaluation and strategic program planning.

Finally, LiST remains unique in examining the impact of malaria interventions within the context of all causes of child mortality. Impacts of malaria interventions on absolute numbers of deaths are influenced by interventions that affect those other causes, as they affect the number of children exposed to malaria mortality. This enables comparisons of malaria interventions with other child survival interventions and focuses attention on opportunities currently being missed for reducing child mortality, by targeting the major causes of child deaths for which effective interventions exist but are not yet implemented to scale.

\section{Limitations}

Limitations of the Spectrum-Malaria and LiST tools, that influence the projection results compared here, have been discussed elsewhere [10-12]. These include that country under-5 malaria mortality data used for the 2015 baseline were themselves model-based and subject to uncertainty and annual re-estimation [1, 40-42]. Further in terms of data inputs, coverage definitions and the assumed baseline and target levels were not necessarily comparable between both models. For ITNs, the relationship between household ownership of $\geq 1$ ITNs (used in LiST) and usage (used by Spectrum-Malaria) is well-characterized by recent multi-country analyses, but this may change as coverage increases and over-allocation to households with already enough ITNs, at the expense of less accessible households who still lack ITNs, increases.

For CMU, both models used coverage estimates for children under- 5 years, but LiST assumed no treatment in older age groups, and considered only treatments within $48 \mathrm{~h}$ of onset of fever, while the CMU coverages used by Spectrum-Malaria ignored timeliness of 
treatment but focused on fevers with RDT-confirmed parasitemia [28]. It is not clear how the two sets of coverage estimates relate, and this uncertainty compounds large uncertainty in effectiveness of CMU.

Malaria treatments in older age groups modeled in Spectrum-Malaria (at the same coverage as for children under-5) but not in LiST, including their dynamic transmission-reducing effects over time, explains why Spectrum-Malaria projected larger long-term impacts of CMU - especially in low-endemic Zambia where high coverage of CMU might, in some of the lower-endemic areas within the country, feasibly drive malaria transmission down to pre-elimination levels. It is hard to say which model is more realistic here, lacking cluster-randomized trials of population-level CMU impacts. The range of impacts between the two models may well capture the real typical magnitude - as well as the uncertainty or variation - in CMU impacts. A more comprehensive, refined comparison between both models of health impacts and cost-effectiveness of CMU should furthermore take into account the required volumes of treatments (across all ages) and other program inputs involved in the respective CMU scale-ups projected. Both models will likely benefit from aligning CMU coverage baseline data, building on progressively improving estimates of effective CMU coverage for parasitologically confirmed malaria to replace current coverage estimates based on child fevers from any cause, that also take into account antimalarial drug availability, quality, patient adherence and compliance, timeliness, caretakers' recall of treatment histories in household surveys [43], and sub-national geographical variation in these determinants of CMU coverage [2, 28, 44]. Such forthcoming refined CMU coverage estimates will also be used for ecological, statistical assessments of historic CMU impacts over space and time in Africa (as recently done for ITNs, IRS and ACT access [2]), which may provide an opportunity to triangulate and validate CMU effectiveness assumptions.

For ITNs, while effectiveness has been fairly precisely estimated for scale-up as occurred over the past decade, and both LiST and Spectrum-Malaria fitted to that, the incremental impacts of ongoing further ITN scale-up may saturate with increasing coverage, which was captured in Spectrum-Malaria but not in LiST. Finally, ITN and IRS impacts will diminish as insecticide resistance develops and spreads. Insecticide resistance was not captured in either model, but should be added as an impact modifier when good-quality standardized data on WHOrecommended resistance indicators become available at country and sub-national resolution [45]. Similarly, CMU effectiveness may be lower in settings with parasitological resistance against the locally used antimalarial drugs [1], an effect modifier not yet captured in either model's impact assumptions.

\section{Implications for program planning}

Going forward, to optimally use LiST and SpectrumMalaria for program and health sector planning, we recommend that program planners use both models in complement. Spectrum-Malaria probably gives the best comprehensive impact projection for ITNs, IRS and CMU -including dynamic transmission effects as they evolve over time and over successive phases of malaria control, and vary with baseline epidemic conditions of each settings. Spectrum-Malaria adds to the arsenal of malaria interventions projected with seasonal malaria chemoprophylaxis (recommended in countries and areas with strongly seasonal malaria, mainly in Western and Sahelian Africa) and with improved management of severe malaria cases. LiST, but not Spectrum-Malaria, models the impact of Intermittent Preventive antimalarial Therapy for pregnant women (IPTp), which benefits women receiving this intervention, and their infants directly. While the population-level impacts of IPTp (which is not known to produce onward dynamic transmission effects at population level) are small compared to transmission-reducing interventions that are the focus of Spectrum-Malaria, IPTp is an essential component of maternal, neonatal and child health care packages, that requires evaluation in the LiST context considering maternal, neonatal and child health outcomes specifically. And only LiST models malaria mortality in the context of other-cause under-five mortality, allowing validation against field program data that typically measure and monitoring all-cause under-five mortality without ascertainment of causes [46]. While Spectrum-Malaria primarily focuses on modeling dynamic interactions among malaria interventions, LiST provides a framework that more easily allows evaluations across a wider set of causes of child deaths with their possible interactions. For example, recent evidence suggests a link between sub-optimal breastfeeding and increased risk of malaria mortality in children under the age of six [47]. Such effects can be captured using the LiST approach, but would not be straightforward to account for in Spectrum-Malaria.

To facilitate the complementary use of LiST and Spectrum-Malaria, modelers should align the baseline country data included in both models, i.e. malaria mortality rates, populations living at risk of Plasmodium falciparum transmission, and recent coverage levels and trends of ITNs, IRS and CMU. To do cost comparison with both models jointly, also their assumed proportions of population living at risk of malaria should be aligned, since these determine the population in need of malaria interventions. Comparison and alignment may be further facilitated if Spectrum-Malaria would compute and display health impacts for pregnant women, as a distinct sub-group of adults. A future way to incorporate in LiST 
the non-linearity in coverage-impact relationships, and saturation and synergy across malaria interventions as illustrated by Spectrum-Malaria, would be for LiST to extend the herd effect c.q. transmission interruption option, that LiST currently allows for modelling of ITNs/ IRS but not yet for malaria treatment.

\section{Conclusion}

In conclusion, Spectrum-Malaria aligns with LiST in the proportional under-5 mortality reduction following ITN scale-up from low (0-20\%) to current/high ITN coverage, as expected given that both models were fitted to the results of the same ITN trials. However, for scale-up from current intermediate coverage levels to universal coverage, Spectrum-Malaria suggests that important country variations and saturation effects in impacts are expected that are not evident from LiST. For CMU, SpectrumMalaria predicts somewhat larger proportional impacts than LiST, which may reflect real long-term dynamic effects, although the precise impact of CMU remains uncertain lacking a controlled-trial-based gold standard. Strategic planners in malaria-endemic African countries can use the two models in a complementary way, within the Spectrum platform, to inform various malaria and health sector strategic planning purposes, including those focusing on population level health beyond child mortality.

\section{Additional files}

Additional file 1: Coverage values assumed in LiST and Spectrum-Malaria impact projections, for Coverage-standardized variants of DR Congo and Zambia (XLSX $11 \mathrm{~kb}$ )

Additional file 2: Relationship between household possession of 1 or more ITNs and population (all ages) sleeping under an ITN, and coverage values used in LiST and Spectrum-Malaria projections for the Democratic Republic of the Congo (DRC) and Zambia. (XLSX $18 \mathrm{~kb}$ )

\section{Abbreviations}

ACT: Artemisinin-based combination therapy; Admin1: First order administrative level geographical units (i.e. states or provinces); CMU: Effective management o uncomplicated malaria cases; DHS: Demographic and Health Survey; DRC: Democratic Republic of the Congo; EIR: Entomological inoculation rate; IRS: Indoor residual spraying; ITN: Insecticide treated mosquito net;

IPTp: Intermittent preventive antimalarial therapy for pregnant women; LiST: Lives Saved Tool; MAP: Malaria Atlas Project; MICS: Multiple Indicator Cluster Survey; MIS: Malaria Indicator Survey; PfPR: Plasmodium falciparum parasite prevalence; RDT: Rapid Diagnostic Test of malaria parasitology; SSA: Sub-Saharan Africa; Swiss TPH: Swiss Tropical and Public Health Institute; WHO: World Health Organization.

\section{Acknowledgements}

The views expressed in this paper are those of the authors and do not necessarily represent the position of Avenir Health, Johns Hopkins University or the Swiss Tropical and Public Health Institute. We thank Ewan Cameron, Daniel Weiss and Samir Bhatt (Oxford University, Malaria Atlas Project) for advice regarding malaria endemicity distributions and indicators, and Richard Cibulskis (WHO Global Malaria Programme) and two anonymous peer reviewers for comments on drafts of the paper. We thank Shufang Zhang (the Global Fund to Fight AIDS, Tuberculosis and Malaria) and Jeremy Lauer (World Health Organization Health Systems Governance and Financing department) for having supported development of the Spectrum-Malaria tool.

\section{Funding}

Avenir Health's contribution was supported by grants from the Global Fund to fight AIDS, Tuberculosis and Malaria; Swiss Tropical and Public Health Institute's contribution was supported by a grant from the World Health Organization's Health Systems Governance and Financing department. The publication costs for all supplement articles were funded by a grant from the Bill \& Melinda Gates Foundation (JHU Grant 115,621, Award Number OPP1084423 for the "Development and Use of the Lives Saved Tool (LiST)").

\section{Availability of data and materials}

The datasets supporting the conclusions of this article are included within the article, its additional files, and the referenced publicly accessible studies.

\section{About this supplement}

This article has been published as part of BMC Public Health Volume 17 Supplement 4, 2017: The Lives Saved Tool in 2017: Updates, Applications, and Future Directions. The full contents of the supplement are available online at https://bmcpublichealth.biomedcentral.com/articles/supplements/ volume-17-supplement-4.

\section{Authors' contributions}

EK, RS and OB designed the study; $\mathrm{MH}, \mathrm{EK}, \mathrm{GM}, \mathrm{RS}$ and NW implemented the analysis; MH compiled WHO and MAP data used as baseline country data for Spectrum-Malaria projections; BW, JS, NW and TS advised interpretation; EK wrote the paper; all authors contributed to interpretation and read and approved the final manuscript.

\section{Ethics approval and consent to participate}

Not applicable.

\section{Consent for publication}

Not applicable.

\section{Competing interests}

The authors declare that they have no competing interests.

\section{Publisher's Note}

Springer Nature remains neutral with regard to jurisdictional claims in published maps and institutional affiliations.

\section{Author details}

${ }^{1}$ Avenir Health, PO box 2100, CH-1211 Geneva, Switzerland. ${ }^{2}$ Avenir Health, 655 Winding Brook Drive, Glastonbury CT-06033, USA. ${ }^{3}$ Epidemiology and Public Health, Swiss Tropical and Public Health Institute, Socinstrasse 57, 4051 Basel, Switzerland. ${ }^{4}$ Epidemiology and Public Health, University of Basel, Basel, Switzerland. ${ }^{5}$ Department of International Health, Institute for International Programs, Johns Hopkins University Bloomberg School of Public Health, 615 N. Wolfe St., Baltimore, MD 21205, USA.

\section{Published: 7 November 2017}

\section{References}

1. World Health Organization. World Malaria Report 2015. Geneva: World Health Organization; 2015. http://who.int/malaria/publications/worldmalaria-report-2015/en/. Accessed 18th Sept 2017.

2. Bhatt S, Weiss DJ, Cameron E, Bisanzio D, Mappin B, Dalrymple U, et al. The effect of malaria control on Plasmodium falciparum in Africa between 2000 and 2015. Nature. 2015;526(7572):207-11.

3. Eisele T, Larsen DA, Walker N, Cibulskis R, Yukich JO, Zikusooka CM, et al. Estimates of child deaths prevented from malaria prevention scale-up in Africa 2001-2010. Malaria J. 2012;93(1):93.

4. Stover J, Ross J. How increased contraceptive use has reduced maternal mortality. Matern Child Health J. 2010;14(5):687-95.

5. Stover J, Johnson P, Zaba B, Zwahlen M, Dabis F, Ekpini RE. The Spectrum projection package: improvements in estimating mortality, ART needs, PMTCT impact and uncertainty bounds. Sex Transm Infect. 2008;84(Suppl 1):i24-30.

6. Stover J, McKinnon R, Winfrey B. Spectrum: a model platform for linking maternal and child survival interventions with AIDS, family planning and demographic projections. Int J Epidemiol. 2010;39(suppl 1):i7-i10. 
7. Houben RM, Lalli M, Sumner T, Hamilton M, Pedrazzoli D, Bonsu F, et al. TIME Impact - a new user-friendly tuberculosis (TB) model to inform TB policy decisions. BMC Med. 2016;14(1):56.

8. Johns Hopkins University Bloomberg School of Public Health. LiST (Lives Saved Tool): An evidence-based decision-making tool for estimating intervention impact. Baltimore 2010. http://idfive.jhsph.edu/centers-andinstitutes-partial/international-health/centers-and-institutes/instituteforinternational-programs/current-projects/lives-saved-tool/index.html. Accessed 18th Sept 2017.

9. Eisele TP, Larsen D, Steketee R. Protective efficacy of interventions for preventing malaria mortality in children in Plasmodium falciparum endemic areas/Modeling the Impact of Scaling up Interventions for Malaria. Int J Epid. 2010;39(Suppl.1):i88-i101.

10. Korenromp EL. Lives saved from malaria prevention in Africa - evidence to sustain cost-effective gains. Malar J. 2012;11:94.

11. McCoy D, Jensen N, Kranzer K, Ferrand RA, Korenromp EL. Methodological and Policy Limitations of Quantifying the Saving of Lives: A Case Study of the Global Fund's Approach. PLoS Med. 2013;10(10):e1001522.

12. Korenromp EL, Hamilton M, Werst E, Mahiané G, Sanders R, Briët OJ, et al. Spectrum-Malaria: a projection tool for health impact assessment and strategic planning for malaria programs in sub-Saharan Africa - Mode structure, methods and assumptions. Glastonbury (CT, USA) \& Geneva (Switzerland): Avenir Health, Swiss Tropical and Public Health Institute, Oxford University Malaria Atlas Project. 2016. Report No.

13. Korenromp EL, Mahiané G, Hamilton M, Pretorius C, Cibulskis R, Lauer J, et al. Malaria intervention scale-up in Africa: statistical effectiveness predictions for health program planning tools, based on dynamic transmission modelling. Malar J. 2016;15(417).

14. Hamilton M, Mahiané G, Werst E, Sanders R, Briët OJ, Smith T, Cibulskis RC, Cameron E, Bhatt S, Weiss DJ, et al. Spectrum-Malaria: a user-friendly projection tool for health impact assessment and strategic planning for malaria programs in sub-Saharan Africa. Malar J. 2017;16(1):68.

15. Smith T, Killeen GF, Maire N, Ross A, Molineaux L, Tediosi F, et al. Mathematical modeling of the impact of malaria vaccines on the clinical epidemiology and natural history of Plasmodium falciparum malaria: Overview. Am J Trop Med Hyg. 2006;75(2 Suppl):1-10.

16. Smith T, Ross A, Maire N, Chitnis N, Studer A, Hardy D, et al. Ensemble modeling of the likely public health impact of a pre-erythrocytic malaria vaccine. PLoS Med. 2012;9(1):e1001157.

17. Lengeler $C$. Insecticide-treated bed nets and curtains for preventing malaria. 2004. Report No.: Contract No.: 3.

18. Tediosi F, Maire N, Smith T, Hutton G, Utzinger J, Ross A, et al. An approach to model the costs and effects of case management of Plasmodium falciparum malaria in sub-saharan Africa. AmJTropMedHyg. 2006;75(2 Suppl):90-103.

19. Cameron E, Battle KE, Bhatt S, Weiss DJ, Bisanzio D, Mappin B, et al. Defining the relationship between infection prevalence and clinical incidence of Plasmodium falciparum malaria. Nat Commun. 2015:6:8170.

20. You D, Hug L, Ejdemyr S, Idele P, Hogan D, Mathers C, et al. Global, regional, and national levels and trends in under- 5 mortality between 1990 and 2015, with scenario-based projections to 2030: a systematic analysis by the UN Inter-agency Group for Child Mortality Estimation. Lancet. 2015;386(10010):2275-86.

21. Thwing J, Eisele TP, Steketee RW. Protective efficacy of malaria case management and intermittent preventive treatment for preventing malaria mortality in children: a systematic review for the Lives Saved Tool. BMC Public Health. 2011;11(Suppl 3):S14.

22. Korenromp EL, Miller J, Cibulskis RE, Cham MK, Alnwick D, Dye C. Monitoring mosquito net coverage for malaria control in Africa: possession versus use by children under 5 years. Tropical Med Int Health. 2003;8(8):693-703.

23. Miller JM, Korenromp EL, Nahlen BL, Steketee RW. Estimating the number of insecticide-treated nets required by African households to reach continent-wide malaria coverage targets. JAMA. 2007;297(20):2241-50.

24. Bhatt S, Weiss DJ, Mappin B, Dalrymple U, Cameron E, Bisanzio D, et al. Coverage and system efficiencies of insecticide-treated nets in Africa from 2000 to 2017. elife. 2015;4(pii:):e09672.

25. MEASURE DHS+. Demographic and Health Surveys (DHS) Calverton, MD, USA: USAID; [cited 201628 July]. Available from: http://measuredhs.com/.

26. UNICEF. Multiple Indicator Cluster Surveys New York City [cited 201628 July 2016]. Available from: http://mics.unicef.org/.

27. Roll Back Malaria, World Health Organization, UNICEF, MEASURE DHS, MEASURE Evaluation, Prevention USCFDCa. Malaria Indicator Survey (MIS)
Toolkit 2005 [cited 201628 July]. Available from: http://malariasurveys.org/ toolkit.cfm.

28. Bennett A, Bisanzio D, Yukich J, Mappin B, Fergus CA, Lynch M, Cibulskis R, Gething PW, Eisele T. Estimates of Africa-wide population coverage of artemisinin-based combination treatment among children under-5 with fever plus Plasmodium falciparum malaria 2000-2015. Lancet Glob Health. 2017:5(4):e418-e427.

29. Otten M, Aregawi M, Were W, Karema C, Medin A, Bekele W, et al. Initial evidence of reduction of malaria cases and deaths in Rwanda and Ethiopia due to rapid scale-up of malaria prevention and treatment. Malar J. 2009:8:14.

30. Aregawi M, Ali AS, Al-mafazy A, Molteni F, Warsame M, Katikiti S, et al. Reductions in malaria and anemia case and death burden to hospitals following scale-up of malaria control in Zanzibar, 1999-2008. Malaria J. 2011;10:46.

31. Korenromp EL, Hosseini M, Newman RD, Cibulskis RE. Progress towards malaria control targets in relation to national malaria programme funding. Malar J. 2013;12:18.

32. Yan I, Korenromp E, Bendavid E. Mortality changes after grants from the Global Fund to Fight AIDS, tuberculosis and malaria: an econometric analysis from 1995 to 2010. BMC Public Health. 2015;15:977.

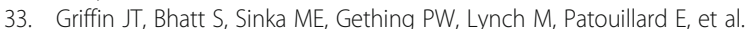
Potential for reduction of burden and local elimination of malaria by reducing Plasmodium falciparum malaria transmission: a mathematical modelling study. Lancet Infect Dis. 2016;16(4):465-72. doi:10.1016/S14733099(15)00423-5.

34. Stuckey EM, Smith T, Chitnis N. Seasonally dependent relationships between indicators of malaria transmission and disease provided by mathematical model simulations. PLoS Comput Biol. 2014;10(9):e1003812.

35. Griffin JT, Hollingsworth TD, Okell LC, Churcher TS, White M, Hinsley W, et al Reducing Plasmodium falciparum malaria transmission in Africa: a model-based evaluation of intervention strategies. PLoS Med. 2010;7:8.

36. Okell LC, Drakeley CJ, Bousema T, Whitty CJ, Ghani AC. Modelling the impact of artemisinin combination therapy and long-acting treatments on malaria transmission intensity. PLoS Med. 2008;5(11):e226. discussion e

37. Penny MA, Galactionova K, Tarantino M, Tanner M, Smith TA. The public health impact of malaria vaccine RTS,S in malaria endemic Africa: country-specific predictions using 18 month follow-up Phase III data and simulation models. BMC Med. 2015;13(1):170.

38. Briët OJ, Penny MA. Repeated mass distributions and continuous distribution of long-lasting insecticidal nets: modelling sustainability of health benefits from mosquito nets, depending on case management. Malar J. 2013;12:401.

39. Pemberton-Ross P, Smith TA, Hodel EM, Kay K, Penny MA. Age-shifting in malaria incidence as a result of induced immunological deficit: a simulation study. Malar J. 2015;14:287.

40. Korenromp EL, Williams BG, Gouws E, Dye C, Snow RW. Measurement of trends in childhood malaria mortality in Africa: an assessment of progress toward targets based on verbal autopsy. Lancet Infect Dis. 2003;3(6):349-58.

41. Cibulskis RE, Bell D, Christophel EM, Hii J, Delacollette C, Bakyaita N, et al. Estimating trends in the burden of malaria at country level. Am J Trop Med Hyg. 2007;77(6 Suppl):133-7.

42. World Health Organization. World Malaria Report 2014. Geneva: World Health Organization; 2014. http://www.who.int/malaria/publications/world_ malaria_report_2014/en/. Accessed 18th Sept 2017.

43. Galactionova K, Tediosi F, De Savigny D, Smith TA, Tanner M. Effective coverage and systems effectiveness for malaria case management in sub-Saharan African countries. PLoS One. 2015;10(5):e0127818.

44. Gething PW, Casey DC, Weiss DJ, Bisanzio D, Bhatt S, Cameron E, et al. Mapping Plasmodium falciparum Mortality in Africa between 1990 and 2015. N Engl J Med. 2016. 2016/10/11. doi:10.1056/NEJMoa1606701.

45. World Health Organization Global Malaria Programme. Global plan for insecticide resistance management in malaria vectors. Geneva: World Health Organization; 2012. http://apps.who.int/iris/bitstream/10665/44846/1/ 9789241564472_eng.pdf. Accessed 18th Sept 2017.

46. Larsen DA, Friberg IK, Eisele TP. Comparison of Lives Saved Tool model child mortality estimates against measured data from vector control studies in sub-Saharan Africa. BMC Public Health. 2011;11(Suppl 3):S34.

47. Victora CG, Bahl R, Barros AJ, Franca GV, Horton S, Krasevec J, et al. Breastfeeding in the 21st century: epidemiology, mechanisms, and lifelong effect. Lancet. 2016;387(10017):475-90.

48. Winfrey W, McKinnon R, Stover J. Methods used in the Lives Saved Tool (LiST). BMC Public Health. 2011;11(Suppl 3):S32. 
49. Ross A, Maire N, Molineaux L, Smith T. An epidemiologic model of severe morbidity and mortality caused by Plasmodium falciparum. Am J Trop Med Hyg. 2006;75(2 Suppl):63-73.

50. Guerra CA, Gikandi PW, Tatem AJ, Noor AM, Smith DL, Hay SI, et al. The limits and intensity of Plasmodium falciparum transmission: implications for malaria control and elimination worldwide. PLoS Med. 2008;5(2):e38.

51. United Nations Dept. of Economic and Social Affairs. population division peaps. World population prospects: the 2012 revision. New York: United Nations Population Division; 2012

Submit your next manuscript to BioMed Central and we will help you at every step:

- We accept pre-submission inquiries

- Our selector tool helps you to find the most relevant journal

- We provide round the clock customer support

- Convenient online submission

- Thorough peer review

- Inclusion in PubMed and all major indexing services

- Maximum visibility for your research

Submit your manuscript at www.biomedcentral.com/submit
Biomed Central 